\title{
Central auditory processing disorder (CAPD) tests in a school-age hearing screening programme - analysis of 76,429 children
}

\author{
Piotr H. Skarzynski ${ }^{1,2,3}$, Andrzej W. Wlodarczyk ${ }^{1,4,5}$, Krzysztof Kochanek ${ }^{1,4}$, Adam Pilka, ${ }^{1,4}$ \\ Wiktor W. Jedrzejczak ${ }^{1,4}$, Lukasz Olszewski ${ }^{1,4}$, Lukasz Bruski ${ }^{3}$, Artur Niedzielski ${ }^{6}$, \\ Henryk Skarzynski ${ }^{1,4}$ \\ 1 Institute of Physiology and Pathology of Hearing, Warsaw, Poland \\ ${ }^{2}$ Clinic Otolaryngology and Rehabilitation, Physiotherapy Faculty, Medical University of Warsaw, Poland \\ ${ }^{3}$ Institute of Sensory Organs, Kajetany, Poland \\ ${ }^{4}$ World Hearing Center, Kajetany, Poland \\ ${ }^{5}$ Institute of Rheumatology, Warsaw, Poland \\ ${ }^{6}$ Otoneurology Lab, Medical University of Lublin, Poland
}

Skarzynski PH, Wlodarczyk AW, Kochanek K, Pilka A, Jedrzejczak WW, Olszewski L, Bruski L, Niedzielski A, Skarzynski H. Central auditory processing disorder (CAPD) tests in a school-age hearing screening programme - analysis of 76,429 children. Ann Agric Environ Med. 2015; 22(1): 90-95. doi: 10.5604/12321966.1141375

\begin{abstract}
Introduction and objective. Hearing disorders among school-age children are a current concern. Continuing studies have been performed in Poland since 2008, and on 2 December 2011 the EU Council adopted Conclusions on the Early Detection and Treatment of Communication Disorders in Children, Including the Use of e-Health Tools and innovative Solutions. The discussion now focuses not only on the efficacy of hearing screening programmes in schoolchildren, but what should be its general aim and what tests it should include? This paper makes the case that it is important to include central auditory processing disorder (CAPD) tests. One such test is the dichotic digits test (DDT). The aim of the presented study was to evaluate the usefulness of the DDT in detecting central hearing disorders in school-age children.

Materials and methods. During hearing screening programmes conducted in Poland in 2008-2010, exactly 235,664 children (7-12-years-old) were screened in 9,325 schools. Of this number, 7,642 were examined using the DDT test for CAPD. Screening programmes were conducted using the Sense Examination Platform.

Results. With the cut-off criterion set at the 5th percentile, results for the DDT applied in a divided attention mode were $11.4 \%$ positive for 7 -year-olds and $11.3 \%$ for 12 -year-olds. In the focused attention mode, the comparable result for 12 -yearolds was $9.7 \%$. There was a clear right ear advantage. In children with positive DDT results, a higher incidence of other disorders, such as dyslexia, was observed.

Conclusions. A test for CAPD should be included in the hearing screening of school-age children. The results of this study form the basis for developing Polish standards in this area.
\end{abstract}

\section{Key words}

central auditory processing disorders, CAPD, hearing screening, hearing loss, school-age children, partial deafness

\section{INTRODUCTION}

Early detection of hearing disorders and prompt therapy helps provide good clinical outcomes and reduces total therapeutic costs. As confirmation, one can point to universal newborn hearing screening programmes successfully implemented in many countries $[1,2]$. One benefit of early diagnosis is successful treatment with a cochlear implant $[3,4]$.

The incidence of problems related to hearing and listening grows with age, leading to the conclusion that in an optimal health care system, screenings should be conducted across all ages in order to implement appropriate therapy at the earliest possible opportunity. In 2008 and 2010, extensive hearing screening programmes were conducted in Poland in which tests were carried out on almost 190,000 children of 7-12 years of age from primary schools in rural areas and small towns (less than 5,000 inhabitants), and on almost

Address for correspondence: Piotr H. Skarzynski, World Hearing Center, Institute of Physiology and Pathology of Hearing, Kajetany, Mokra 17, 05-830 Nadarzyn, Poland E-mail: intercollab@ifps.org.pl

Received: 15 March 2013; Accepted: 03 January 2014
50,000 12-year-olds in larger cities [5,6]. These programmes inspired the 'EU Council Conclusions on Early Detection and Treatment of Communication Disorders in Children, Including the Use of e-Health Tools and Innovative Solutions' of 2 December 2011 (2011/C361/04) [7].

The incidence of hearing loss in schoolchildren is between $10-15 \%$, while central auditory processing disorders (CAPDs) are estimated to affect an additional $2-7 \%$ [8]. Both hearing loss and CAPDs have a negative impact on a child's development in terms of communication, learning, and social skills. Undetected and untreated, CAPD adversely affects the child's quality of life [9]. CAPDs can coexist with speech disorders, dyslexia, and other problems. Many arguments can be made for the efficacy of therapy for CAPD, especially when conducted early, and for the positive impact of auditory training on acquiring reading and writing skills $[10,11]$.

The usefulness of CAPD screening has been much discussed $[12,13]$. In the 1980s, a battery of screening tests called 'SCAN' was developed in the USA which included dichotic listening, speech understanding in noise, and filtered 
speech tests. Studies have demonstrated that, of the three, the dichotic listening test based on competing sentences has the highest sensitivity and specificity [14]. Other CAPD screening tools, which include DDT, are the 'MAPA' battery of tests, including several for detecting anomalies in higher auditory function [15], and the 'DSTP' battery of tests which assess dysfunctions in perceiving acoustic, phonological, and language characteristics [16]. To-date, there have been no large-scale screening programmes conducted using the above tests; they have been limited to groups of 81 children (MAPA) and 509 children (DSTP) [17].

The DDT is often used in clinical practice as it requires little in terms of language skill and memory load and is easy to perform. Because of these features it is widely recommended and considered the most valuable of the CAPD screening tests [18].

In 2007, a Polish version of the DDT was developed at the Institute of Physiology and Pathology of Hearing in Warsaw. Tests of its practical application as a CAPD screening test, in accordance with ASHA recommendations [15], followed in $2008-2010$. The aim of this study was to evaluate the usefulness of DDT for detecting CAPDs in school-age children.

\section{MATERIALS AND METHOD}

Hearing screening programmes were conducted in 2008 and 2010 in 9,325 primary schools in Poland and included 235,664 children 7 - 12 y.o. - 48.7\% girls and $51.3 \%$ boys. Screening focused primarily on first graders $(75.7 \%)$. Older children, $8-12$ y.o. (24.3\%), were mostly referred for screening tests by parents or teachers who had observed hearing problems. In some local programmes, e.g. in Warsaw, yearly screening tests encompassed the entire population of 12-year-olds.

The incidence of conductive or sensorineural hearing loss was assessed in all children, while CAPDs were tested for in 76,429 children $(32.4 \%)$, of which $45.5 \%$ were 7 y.o. and $44.9 \%$ were 12 y.o.

The study used the 'Sense Examination Platform' device (Institute of Sensory Organs, Warsaw, Poland). The instrument meets the requirements of European norm EN 60645-1 for Class 4 audiometers and carries a CE mark for medical devices. Its audiometric range is from 250 $8000 \mathrm{~Hz}$, and sound output from $0-80 \mathrm{~dB}$ HL (resolution $5 \mathrm{~dB}$ ). The device is equipped with headphones (Sennheiser HAD 200) which conform to IEC 60645-1. It is provided with screening tests for CAPD as well as for sight and speech, with questionnaires adjusted for each screening programme.

In hearing screening aimed at detecting conductive or sensorineural hearing loss, in accordance with ASHA requirements [15], hearing thresholds were evaluated manually using pure tone audiometry (called the pure tone screening test, PTS). Testing was carried put using tone bursts at $250,500,1,000,2,000,4,000$, and $8,000 \mathrm{~Hz}$, and in certain cases also at $6,000 \mathrm{~Hz}$. The criterion for a clear (positive) result was a hearing threshold at 1,000, 2,000, 4,000, and $8,000 \mathrm{~Hz}>20 \mathrm{~dB}$ HL.

For CAPDs, screening was carried out using a Polish version of the DDT, comprising 20 pairs of digits from $1-9$ presented at $60 \mathrm{~dB}$ HL. In 7-year-olds, DDT was performed in the divided attention mode, in which the child's task was to repeat all heard digits. In 12-year- olds, DDT was performed both in divided attention and in focused attention (child had to repeat the digits it heard in one specified ear) for each ear separately.

Parents of screened children were asked to complete a questionnaire, in which the following questions concerned problems and symptoms related to CAPD:

1. Does your child demonstrate any problems relating to hearing?

2. Is your child's speech development delayed of disturbed?

3. Does your child often ask you to repeat sentences?

4. Does your child have problems with social contacts?

5. Does your child have decreased attention focus?

6. Does your child have dyslexia?

7. Does your child have problems with learning foreign languages?

8. Does your child have frequent otitis?

Parents of 7-year-olds were asked only questions 1,2 , 3,4 , and 5 . There were 2 additional questions with multiple choice responses:

9. Does your child ask you to repeat questions? Responses: never, seldom, often, very often.

10. What is your child's school performance? Responses: very poor, poor, good, very good.

Screening tests were performed by trained personnel in quiet rooms in schools during lesson times, but not during breaks. The results of hearing tests and the questionnaire were collected in a database connected to the central screening management system, SZOK, where they underwent an automatic analysis process.

Test results were automatically analyzed for reliability by noting the start time, activity time, and end time of each measurement. The DDT test was performed directly after the PTS. Tests performed in too short a time were not included in the analysis. Correct performance of the DDT and PTS required that the tests were performed in a time of at least 1.5 minutes in children 7 y.o., 40 seconds in 12 y.o. children in focused attention mode, and 70 seconds in children 12 y.o. in divided attention mode. Analysis excluded results from schools where the acoustic conditions under which the tests were conducted did not guarantee their accuracy.

After each test, the researcher had to respond to questions about acoustic conditions. If the automatic system flagged a problem, a specialist physician decided about the need for further diagnostic examinations.

The large number of screened children enabled a DDT norm to be determined based on the epidemiology analyses and calculating the percentage of incorrect DDT results (children who obtained results below the 5th percentile).

Assessment of the relation between the DDT results and questionnaire data was performed based on contingency tables, using chi-squared tests for analysis of correlation between variables. Strength of the relationship between variables was determined based on Cramér's V and tetrachoric correlations. 


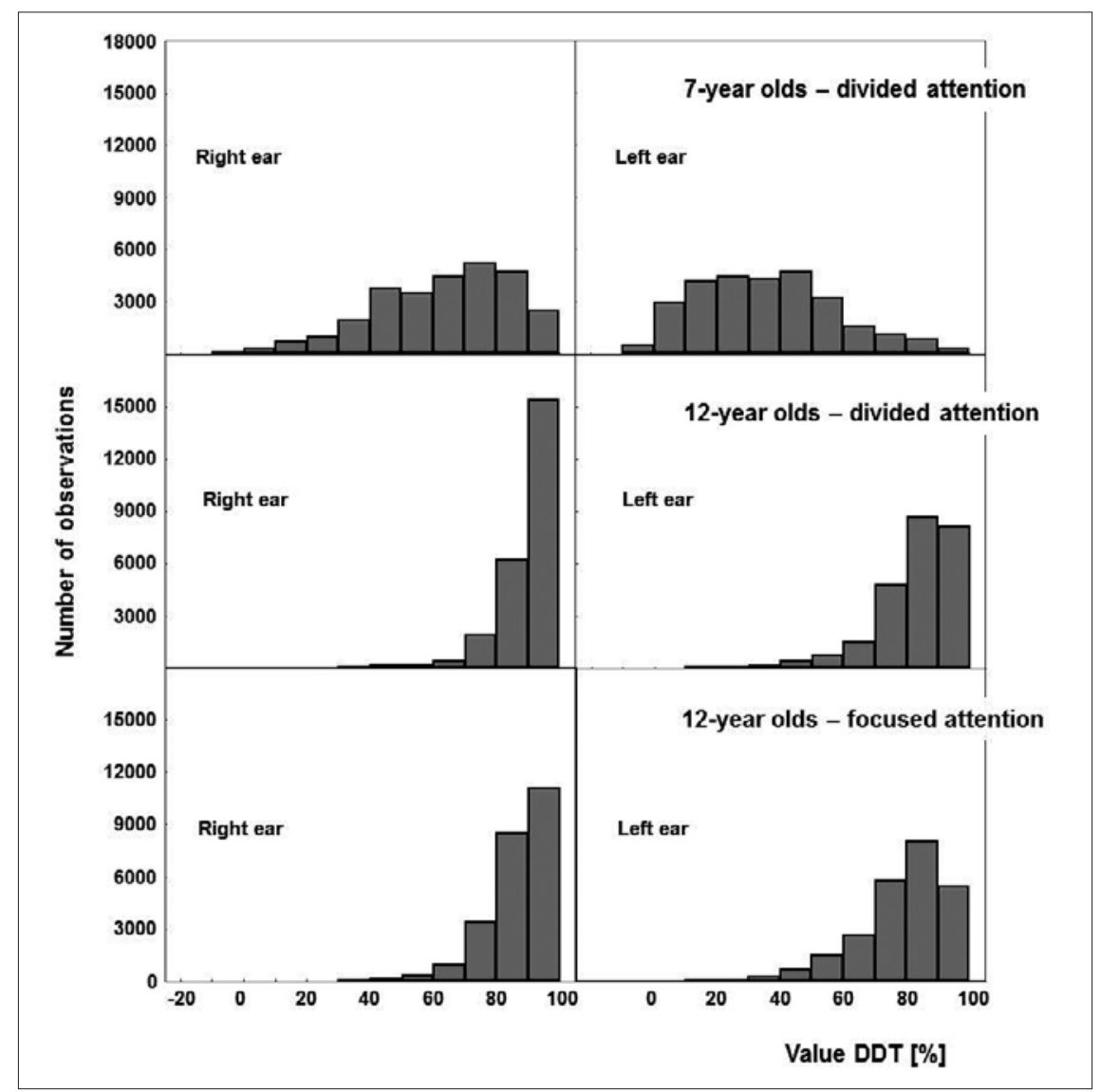

Figure 1. Histograms of DDT results in the right ear (first column) and left ear (second column) in the divided attention mode for 7 and 12-year-olds, and in focused attention mode for 12-year-olds

\section{RESULTS}

The proportion of boys to girls was the same as in the entire population, yet the percentage of incorrect results was slightly higher in boys (7-year-olds - $13.3 \%$ in boys, $12.7 \%$ in girls; 12 -year-olds $-9.3 \%$ in boys, $8.7 \%$ in girls).

Evaluation of the DDT border parameters. Figure 1 shows the distribution of DDT values for the right and the left ears in both age groups.

In the group of 7-year-olds, a large difference was observed between the results obtained with the right and left ear, demonstrating a right ear advantage REA [20]. The REA value in this group was $27.6 \%$ with a standard deviation (SD) of $40 \%$. In the group of 12 -year-olds, the REA value was $6.9 \%$ (SD 13.0\%) in the divided attention mode, and in the focused attention mode the comparable figure was $7.8 \%$ (SD 13.3\%).

DDT distribution parameters for the group of 28,333 7-year-olds and 24,570 12-year-olds with negative hearing screening results are shown in Table I The range of the norm was taken to be above the 5 th percentile.

Based on these criteria, the frequency of incorrect DDT results in both age groups was determined. The incidence of CAPD in 12 y.o. children took into account the DDT results in both the divided and focused attention conditions. The children were also separated into a group who had negative DDT results in both attention modes, and another group in which the DDT results were incorrect in at least one mode (Tab. 2)
Applying the cut-off criterion at the 5th percentile, the frequency of positive results for DDT in the divided attention group of 7-year-olds was $11.4 \%$ and in the group of 12 -yearolds $11.3 \%$. Incorrect DDT results for the focused attention test were observed in $9.7 \%$ of 12 -year-olds. Incorrect DDT results for both attention modes were observed in 1,054 children $(4.3 \%)$, and for at least one of the two attention modes 4,094 children (16.7\%).

Comparison of the questionnaire data for children diagnosed with and without CAPD (according to the DDT test) was performed for groups of 22,170 12-year-olds and 22,307 7-year-olds who had negative PTS results.

In the group of 7-year-olds there was no observed relationship (very low tetrachoric correlation quotient) between the DDT result and the incidence of hearing problems and attention problems reported by parents.

In the group of 12-year-olds there were statistically significant relationships between the DDT results and different aspects of the child's hearing function, as noted in the questionnaire. In children with incorrect DDT results, parents noted significantly more hearing problems, attention deficit problems, difficulties in social contacts, and difficulties in making friends compared with their peers. In children with incorrect DDT results, the incidence of dyslexia and problems in learning foreign languages was nearly twice as high as in children with correct DDT results. The strongest relationships were observed in children with incorrect DDT results in both attention modes (Tab. 3). However, there was no observed relationship between past otitis media and an incorrect DDT result. 
Table 1. Mean, median, standard deviation (SD), and DDT values for 5th and 10th percentile in children 7 and 12 y.o.

\begin{tabular}{|c|c|c|c|c|c|c|}
\hline \multicolumn{7}{|c|}{7 y.o. divided attention } \\
\hline & $\mathrm{N}$ & Mean & Median & 5 th percentile & 10th percentile & SD \\
\hline right ear & 28,333 & $64.8 \%$ & $68 \%$ & $25 \%$ & $37 \%$ & $21.3 \%$ \\
\hline left ear & 28,333 & $37.2 \%$ & $35 \%$ & $5 \%$ & $10 \%$ & $21.9 \%$ \\
\hline \multicolumn{7}{|c|}{12 y.o. divided attention } \\
\hline & $\mathrm{N}$ & Mean & Median & 5th percentile & 10th percentile & SD \\
\hline left ear & 24,570 & $81.4 \%$ & $85 \%$ & $55 \%$ & $60 \%$ & $14.0 \%$ \\
\hline \multicolumn{7}{|c|}{12 y.o. focused attention } \\
\hline & $\mathrm{N}$ & Mean & Median & 5th percentile & 10th percentile & SD \\
\hline right ear & 24,570 & $92.3 \%$ & $95 \%$ & $75 \%$ & $80 \%$ & $10.2 \%$ \\
\hline left ear & 24,570 & $85.4 \%$ & $90 \%$ & $60 \%$ & $70 \%$ & $12.9 \%$ \\
\hline
\end{tabular}

Table 2. Number and percentage of children with incorrect DDT results

\begin{tabular}{|c|c|c|c|}
\hline \multicolumn{4}{|c|}{7 y.o. - incorrect DDT in divided attention mode } \\
\hline border value & Gender & number of incorrect results & percentage of incorrect results \\
\hline & girls & 1,658 & $11.4 \%$ \\
\hline \multirow[t]{2}{*}{ 5th percentile } & boys & 1,581 & $11.5 \%$ \\
\hline & total & 3,239 & $11.4 \%$ \\
\hline \multicolumn{4}{|c|}{12 y.o. - incorrect DDT in divided attention mode } \\
\hline & Girls & 1,217 & $9.8 \%$ \\
\hline \multirow[t]{2}{*}{ 5th percentile } & Boys & 1,549 & $12.8 \%$ \\
\hline & Total & 2,766 & $11.3 \%$ \\
\hline \multicolumn{4}{|c|}{12 y.o. - incorrect DDT in focused attention mode } \\
\hline \multirow[t]{2}{*}{ border value } & Gender & number of incorrect results & percentage of incorrect results \\
\hline & Girls & 938 & $7.6 \%$ \\
\hline \multirow[t]{2}{*}{5 th percentile } & Boys & 1,444 & $11.9 \%$ \\
\hline & Total & 2,382 & $9.7 \%$ \\
\hline \multicolumn{4}{|c|}{12 y.o. - incorrect DDT in focused and divided attention modes } \\
\hline \multirow[t]{2}{*}{ border value } & Gender & number of incorrect results & percentage of incorrect results \\
\hline & Girls & 389 & $3.1 \%$ \\
\hline \multirow[t]{2}{*}{ 5th percentile } & Boys & 665 & $5.5 \%$ \\
\hline & Total & 1,054 & $4.3 \%$ \\
\hline \multicolumn{4}{|c|}{12 y.o. - incorrect DDT in focused or divided attention modes } \\
\hline \multirow[t]{2}{*}{ border value } & Gender & number of incorrect results & percentage of incorrect results \\
\hline & Girls & 1,766 & $14.2 \%$ \\
\hline \multirow[t]{2}{*}{ 5th percentile } & Boys & 2,328 & $19.2 \%$ \\
\hline & Total & 4,094 & $16.7 \%$ \\
\hline
\end{tabular}

Table 3. Incidence of an affirmative answer to the respective questionnaire item (indicating the existence of a problem) compared to DDT results in both attention modes in 12-year olds

\begin{tabular}{|c|c|c|c|c|c|c|}
\hline & \multirow[b]{2}{*}{ DDT normal } & \multicolumn{3}{|c|}{ DDT incorrect } & \multirow[b]{2}{*}{$p$ value } & \multirow[b]{2}{*}{ Cramér's V } \\
\hline & & focused attention & divided attention & $\begin{array}{l}\text { both attention } \\
\text { modes }\end{array}$ & & \\
\hline Child had delayed speech development & $7.2 \%$ & $13.2 \%$ & $13.9 \%$ & $24.3 \%$ & $10^{-7}$ & 0.14 \\
\hline Child has decreased attention focus & $31.7 \%$ & $37.0 \%$ & $40.8 \%$ & $50.9 \%$ & $10^{-7}$ & 0.10 \\
\hline Child has dyslexia & $24.3 \%$ & $29.8 \%$ & $39.6 \%$ & $45.8 \%$ & $10^{-7}$ & 0.13 \\
\hline $\begin{array}{l}\text { Child has problems with learning foreign } \\
\text { languages }\end{array}$ & $23.3 \%$ & $29.7 \%$ & $42.3 \%$ & $47.3 \%$ & $10^{-7}$ & 0.16 \\
\hline Child had otitis media & $42.0 \%$ & $41.43 \%$ & $38.5 \%$ & $40.3 \%$ & 0.0401 & 0.02 \\
\hline $\begin{array}{l}\text { Child has problems with social contacts or } \\
\text { making friends }\end{array}$ & $7.9 \%$ & $11.7 \%$ & $12.0 \%$ & $18.2 \%$ & $10^{-7}$ & 0.08 \\
\hline
\end{tabular}


Table 4. Prevalence of particular responses in 12-year-olds with correct and incorrect DDT results for two attention modes

\begin{tabular}{|c|c|c|c|c|c|c|c|}
\hline Question & Response & normal DDT & $\begin{array}{c}\text { incorrect DDT } \\
\text { (focused attention) }\end{array}$ & $\begin{array}{c}\text { incorrect DDT } \\
\text { (divided attention) }\end{array}$ & $\begin{array}{c}\text { incorrect DDT } \\
\text { (both attention } \\
\text { modes) }\end{array}$ & $p$ value & Cramér's V \\
\hline \multirow{4}{*}{$\begin{array}{l}\text { Child asks for repetition of } \\
\text { the question or information }\end{array}$} & never & $17.1 \%$ & $13.8 \%$ & $14.7 \%$ & $12.5 \%$ & \multirow{4}{*}{$10^{-7}$} & \multirow{4}{*}{0.06} \\
\hline & seldom & $71.9 \%$ & $71.4 \%$ & $72.0 \%$ & $66.5 \%$ & & \\
\hline & often & $10.2 \%$ & $13.5 \%$ & $11.9 \%$ & $18.2 \%$ & & \\
\hline & very often & $0.8 \%$ & $1.3 \%$ & $1.4 \%$ & $2.8 \%$ & & \\
\hline \multirow{4}{*}{ Child's school grades } & very good & $36.6 \%$ & $27.7 \%$ & $19.7 \%$ & $13.4 \%$ & \multirow{4}{*}{$10^{-7}$} & \multirow{4}{*}{0.10} \\
\hline & good & $48.5 \%$ & $47.2 \%$ & $52.6 \%$ & $49.5 \%$ & & \\
\hline & poor & $13.9 \%$ & $23.6 \%$ & $24.9 \%$ & $33.0 \%$ & & \\
\hline & very poor & $1.00 \%$ & $1.6 \%$ & $2.8 \%$ & $4.0 \%$ & & \\
\hline
\end{tabular}

Table 5. Values of DDT parameters obtained in the present study (in Polish) and norms used previously in English

\begin{tabular}{lcccc}
\hline \multirow{2}{*}{ Age group } & \multicolumn{2}{c}{ DDT in Polish } & \multicolumn{2}{c}{ DDT in English } \\
\cline { 2 - 5 } & right ear & left ear & right ear & left ear \\
\hline 7 y.o. & $5 \%$ & $25 \%$ & $55 \%$ & $70 \%$ \\
\hline 12 y.o. & $55 \%$ & $70 \%$ & $88 \%$ & $90 \%$ \\
\hline
\end{tabular}

Analysis of responses to the 2 questions that carried graded replies (Tab. 4) showed that children with incorrect DDT results more frequently required information to be repeated in order to understand it. Children with incorrect DDT results had a significantly higher percentage of poor school grades compared to children with correct DDT results. The percentage of very good school grades was twice as low in children with incorrect DDT results, in both attention modes, compared to children with correct DDT results.

\section{DISCUSSION}

The large material of the presented study enables norms for the Polish version of the DDT to be developed. Comparison of the norms determined in this study (for divided attention using the 5th percentile criterion) with norms for the English language equivalent [19] determined using a criterion of 2 standard deviations below the mean $(-2 \mathrm{SD})$ is shown in Table 5.

The large disparity in Polish and English norms confirms the necessity to set norms in each language.

Right ear advantage (REA) for speech stimuli, a wellknown phenomenon that indicates maturation of the auditory system, was observed [20].

In focused attention mode, DDT assesses separation between ears, that is the ability to suppress the signal from one ear and one brain hemisphere, while in the divided attention mode it assesses the integration of information from both ears. While the focused attention results depend on the maturity of the corpus callosum, in the divided attention mode they depend more on correct auditory attention and memory [21]. Based on the results of this study, it can be concluded that both the focused and divided attention DDTs can identify children with a high incidence of symptoms characteristic of CAPDs. However, the highest predictive value is achieved when the DDT is incorrect for both attention modes.
In the presented material, DDT was demonstrated to be unreliable in 7-year-olds. This may be because these children had been attending school for only a few months, giving rise to feelings of less self-confidence and more insecurity than needed to give full attention to the test. At this age, the maturity of the auditory system is also very different: 7 years is the lowest age for which for DDT norms exist [22]. Moreover, examinations should not be conducted in months when there is increased risk of upper respiratory tract infections [23].

In the presented material, incorrect DDT results occurred in $37 \%$ of children who had dyslexia. Other studies have shown that the incidence of CAPDs in children with dyslexia is about $50 \%[10,24]$.

In the tested group of children with an incorrect DDT result, $10.7 \%$ had delayed language development and $49.5 \%$ had dyslexia. The presented study material confirms the close relationship between the incidence of speech dysfunctions and CAPDs. Other studies have also demonstrated children with suspected CAPD are also more likely to have speech disorders $[12,25]$.

The present diagnostic standard for CAPD is performing a battery of tests, each one assessing a different central auditory function. When performing only the DDT, only those children with CAPD who have dysfunction related to interaural separation and integration can be detected. Evaluation of the value of DDT as a screening test for CAPD is not easy, as there are no detailed data on how many children with CAPD have problems with BI/BS (binaural integration/ binaural separation), and how many have problems with auditory pattern temporal ordering (APTO) or monaural separation closure (MSC). In order to fully confirm the efficacy of the DDT, and to determine its sensitivity and specificity, it will be necessary to conduct a control study using tests for central auditory functions on a random sample of children from the screening group [12, 15, 25].

It seems clear, therefore, that at the present level of development of audiology in Poland, the optimal model of CAPD screening should be 2-stage: the first screen should be a specialized audiological questionnaire, and the second a CAPD test battery which should certainly include the DDT test. 


\section{CONCLUSIONS}

Using a computer makes it possible to comprehensively screen for conductive and sensorineural hearing loss, as well as for central auditory disorders. Combining an audiological questionnaire and the DDT makes it possible to detect, even in a school environment, children with coexisting central auditory disorders and other developmental disorders.

Analysis of the questionnaire data has shown that hearing difficulties, speech dysfunctions, and school problems occur significantly more often in children with hearing loss; they also occur more frequently in children with normal hearing thresholds and CAPD.

\section{Acknowledgement}

The authors express their thanks to Andrew Bell for comments on an earlier version of this article.

\section{REFERENCES}

1. Ciorba A, Hatzopoulos S, Camurri L, Negossi L, Rossi M, Cosso D, et al. Neonatal newborn hearing screening: four years' experience at Ferrara University Hospital (CHEAP project): part 1. Acta Otorhinolaryngol Ital. 2007; 27(1): 10-16.

2. Hatzopoulos S, Qirjazi B, Martini A. Neonatal hearing screening in Albania: results from an ongoing universal screening program. Int J Audiol. 2007 Apr;46(4):176-82.

3. Skarzynski H, Matusiak M, Lorens A, Skarzynski PH, Piotrowska A. Partial deafness treatment in children by using Cochlear SRA electrode - round window surgical technique and evaluation by comparison of preservation of residual hearing and insertion depth angle. 10th European Symposium on Paediatric Cochlear Implantation (ESPCI), Athens, Greece, Medimond 2011. pp.115-120.

4. Skarzynski, H, Porowski M, Mrowka M, Barylyak, R, Szkielkowska A, Mlotkowka-Klimek P, et al. Cochlear implantation in congenital middle and inner ear malformations in children. 10th European Symposium on Paediatric Cochlear Implantation (ESPCI), Athens, Greece, Medimond 2011.pp.93-95.

5. Skarzynski PH, Kochanek K, Skarzynski H, Senderski A, Wysocki J, Szkielkowska A, et al. Hearing screening program in school-age children in western Poland. Int Adv Otol. 2011; 7(2): 194-200.

6. Skarzynski H, Kochanek K, Senderski A, Skarzynski PH, Ludwikowski M, Kopaczewski M. Organization of the hearing screening examinations in Polish schools in rural areas and small towns. Cochlear Implants International 2010, 11(Suppl 1): 143-147.
7. Skarzynski H, Piotrowska A. Screening for pre-school and schoolage hearing problems: European Consensus Statement. Int J Pediatr Otorhinolaryngol. 2012; 76(1): 120-121.

8. Musiek FE, Gollegly K, Lamb L, Lamb P. Selected issues in screening for central auditory processing dysfunction. Seminars in Hearing 1990; 11: 372-384.

9. Musiek FE, Gollegly KM, Kibbe KS, Verkest-Lenz SB. Proposed screening test for central auditory disorders: follow-up on the dichotic digits test. Am J Otol. 1991; 12(2): 109-113.

10. Wlodarczyk E, Szkielkowska A, Skarzynski H, Pilka A. Assessment of the efficiency of the auditory training in children with dyslalia and auditory processing disorders. Otolaryngol Pol. 2011;65(5): 339-344.

11. Schochat E, Musiek FE, Alonso R, Ogata J. Effect of auditory training on the middle latency response in children with (central) auditory processing disorder. Braz J Med Biol Res. 2010; 43(8): 777-785.

12. Chermak GD, Silva ME, Nye J, Hasbrouck J, Musiek FE. An update on professional education and clinical practices in central auditory processing. J Am Acad Audiol. 2007; 18(5): 428-452.

13. Katz J, Tillery KL. Can central auditory processing tests resist supramodal influences? Am J Audiol. 2005; 14(2): 124-127.

14. Amos NE, Humes LE. SCAN test-retest reliability for first- and thirdgrade children. J Speech Lang Hear Res. 1998; 41(4): 834-845.

15. Schow RL, Seikel JA, Chermak GD, Berent M. Central auditory processes and test measures: ASHA 1996 revisited. Am J Audiol. 2000; 9(2): 63-68.

16. Richard GJ, Ferre JM. Differential screening test for processing. East Moline, IL, LinguiSystems Inc. 2006.

17. Shiffman JM. Accuracy of CAPD screening, a longitudinal study. Master's thesis. Pocatello, Idaho State University, 1999.

18. Jerger J, Musiek F. Report of the consensus conference on the diagnosis of auditory processing disorders in school-aged children. J Am Acad Audiol. 2000; 11(9): 467-474.

19. Bellis TJ, Ross J. Performance of normal adults and children on central auditory diagnostic tests and their corresponding visual analogs. J Am Acad Audiol. 2011; 22(8): 491-500.

20. Berlin CI, Hughes LF, Lowe-Bell SS, Berlin HL. Dichotic right ear advantage in children 5 to 13 . Cortex. 1973; 9(4): 394-402.

21. Chmiel R, Jerger J. Hearing aid use, central auditory disorder, and hearing handicap in elderly persons. J Am Acad Audiol. 1996; 7(3): 190-202.

22. Musiek FE. Assessment of central auditory dysfunction: the dichotic digit test revisited. Ear Hear. 1983; 4(2): 79-83.

23. Sliwa L, Hatzopoulos S, Kochanek K, Pilka A, Senderski A, Skarzynski $\mathrm{PH}$. A comparison of audiometric and objective methods in hearing screening of school children. A preliminary study. Int J Pediatr Otorhinolaryngol. 2011; 75(4): 483-438.

24. King WM, Lombardino LJ, Crandell CC, Leonard CM. Comorbid auditory processing disorder in developmental dyslexia. Ear Hear. 2003; 24(5): 448-556.

25. Sharma M, Purdy SC, Kelly AS. Comorbidity of auditory processing, language, and reading disorders. J Speech Lang Hear Res. 2009; 52(3): $706-722$. 